\title{
ZeitschriftfürangewandteChemie.
}

XIX. Jahrgang.

Heft 26.

29. Juni 1908.

Allelnige Annahme von Inseraten beỉ den Annoncenexpeditionen von Angust Scherl G. m. b. H. und Daube \& Co., G. m. b. H., Berlin SW. 12, Zimmerstr. 37-41

sowie in deren Filialen: Bremen, Obernstr. 16. Brenlan, Schweidnitzerstr. 11. Dreeden, Seestr. 1. EIberfold, Herzogstr, 38. Frenkfurt a. M., Keiserstr. 10. Hamburr, Alter Wall 76. Hannover, Geosrgtr. 39. Kaseel, Obert Königstr. 27. Koln a. Rh., Hohestr. 145. Leipalg, Petersstr. 19, I. Mardeburo, Breiteweg 184, I. Munohen. Kaufingerstrafe 25 (Domfreiheit). Nurnbery, Kaiserstrase Geke Floischbrticke. Btuttgart, Konigitr. 11, l Kaufingerstrafe 25 (Domfreiheit). Nurnbery, Kaiserstrabe Ecke

Dor Insertionspreis betrăgt pro mm Höhe bel $45 \mathrm{~mm}$ Breite (3 gespalten) 15 Pfennige, auf den beider auBeren Umachlagseiten 20 Pfennige. Bei Wiederholungen tritt enteprechender Rabatt eín. Beilagen werden pra
1000 Sttuck mit 8.- M fur 5 Gramm Gewicht berechnet; fur schwere Beilagen tritt besondere Vereinbarung ein.

I N H A L T:

H. Bucherer: Die Teerfarbenchemie in den Jahren 1904 und 19051169

S. Littmann: Poktische Beiträge zur Bleikammertheorie 1177.

E. Hartman n v. F. B enker: Mechanische Röstöfen beim Bleikammerprozeßs (Schluß) 1188

H. Weil: Unterricht in Theorie und Praxis des Patentgesetzes für Chemiker und Ingenieure auf deutschen Hoch. sihulen 1195 .

H. Ost: Zur Geschichte.der Strontianentzuckerung 1196.

H. M a s t b a m: Über die Beurteilnng der Branntweine 1197.

Referate :

Analytische Chemie, Laboratoriumsapparate und allgemeine Laboratoriumsverfahren 1198; -- Chemie der Nahrungs und Genußmitiel, Wasserversorgung und Hrgiene 1204.

\section{Wirtschaftlioh-gewerblicher Teil:}

Tagevgeschichtliche und IIandelsruudschau: Neu-York; - Das Hall-Patent für die Erzeugung von Aluminium er Joschen: -... Amstadt: Gewerbeakademie; - Berlin; - Griefheim 1207; - Stuttgart; - Zuschrift von Reh \& Co.: Die Asphaltlager bei San Valentino. Italien; - Handelsnotizen 1208; - Dividenden; - Aus anderen Vereinen: Natur forschende Gesellschaft in Basfl: Prof. Fichter: Über elektrolytische Reduktionen; ders.: Über Schwefelfarbstoffe 1210; - I'ersonalnotizen; - Neue Bücher; - Bücherbesprechungen 1211; - Patentlisten 1218.

Berichtigungen 1216.

\section{Die Teerfarbenchemie in den Jahren 1904 und 1905.}

Von Hans Bucherer.

(Eingeg. d. 11.4. 1006.)

Auch in den letzten Jahren lassen die finanziellen Ergebnisse der mit stetig wachsenden Kapitalien arbeitenden großen Unternehmungen nicht den geringsten Zweifel darüber, daß die Teerfarbenindustrie, und besonders die deutsche, sich eines durchaus blühenden Zustandes erfreut. Anders ist die Sachlage, wenn es sich um die Bewertung des wissenschaftlichen und des damit eng zusammenhängenden technischen Fortschrittes handelt, den uns die jüngst vergangene Zeit gebracht hat. Es ist mehrfach die Ansicht geäußert worden, daß die Teerfarbenindustrie ihren Höhepunkt überschritten habe. Einer solchen Auffassung läßt sich in dieser Form wohl kaum beipflichten, wenn man darunter nicht ctwa verstehen will, daß sich die Fntwicklung in einem gegen früher etwas gemäßigten Tempo vollzieht, oder um die Verhältnisse durch eine andere uns geläufige Form zu verdeutlichen : Der Anstieg der Kurve, duroh welche die Entwicklung der Teerfarbenindustrie zum Ausdruck gebracht wird, ist etwas weniger steil geworden, ohne daB die Kurve jedoch bisher ihr Maximum orteicht hat. Es bedarf nur eines Hinblickes auf dic engen Beziehungen zwischen Farben- und Textilindustrie, um ohne weiteres zu erkennen, daB die Zukunft der Farbenfabriken eine durchaus gesicherte ist. Die Auf- schließung weiterer Absatzgebiete für den allgemeinen Welthandel wird zweifellos in hohem Maße dem Textilgewerbe und durch dieses der Teerfarbenindustrie zugute kommen, abgesehen davon, daß auch die unmittelbare Ausfuhr von Teerfarbstoffen nach fremden, überseeischen Ländern in der gegenwärtigen Zeit eine große Rolle spielt. Viel schwieriger würde es sein, voraussagen zu wollen, wie sich der Wettbewerb der einzelnen Länder untereinander gestalten wird. Daß Deutschland mit seiner Teerfarbenindustrie zurzeit einen nicht unbeträchtlichen Vorsprung vor den anderen Ländern besitzt, das lchren die statistischen Angaben über die Zahl der Fabriken und der in ihnen beschäftigten Chemiker und Arbeiter, sowie der in ihnen angelegten Kapitalien und der erzeugten Produkte. Es muß aber darauf hingewiesen werden, daß alle diejenigen Iänder, die eine hochentwickelte Textilindustrie aufzuweisen haben, wie England, die Vereinigten Staaten und Frankreich, eine der allerwichtigsten Voraussetzungen für das Gedeihen der Farbenindustrie erfüllen, und daß daher die Möglichkeit nicht ausgeschlossen erscheint, daß Deutschland seine führende Rolle verliert, umsomehr als von den beiden wichtigsten Rohstoffen, Baumwolle und Wolle (von der Seide also ganz abgesehen), Deutschland bisher keinen in nennenswertem Umfange selbst crzeugt. Die Vereinigten Staaten bringen aber den größten Teil des Weltbedarfs an Baumwolle hervor, und England besitzt wonigstens in seinen Kolonien Gebiete, die zum Teil es schon jetzt mit den für seine Industrie erforderlichen Rohmaterialien ver- 
sorgen, teils des Augenblicks harren, in dem der entschiedene Wille, von den Launen sogenannter amerikanischer Baumwollkönige unabhängig zu sein, - ebenso wie in Deutsch-Togo - den Anbau der Baumwolle veranlassen wird.

Deutschland hat in mehr als einem Falle das, was die Natur seinem Boden versagt hat, durch die Intensität wissenschaftlicher Forschung und mit Hilfe einer überlegenen Technik auszugleichen verstanden. Die Gebiete der Wissenschaft und Technik sind aber auch anderen Nationen leicht zugänglich, zumal bei dem heutigen lebhaften Verkehr der Kulturvölker untereinander. Insofern also scheint der gegenwärtige Zustand keine sichere Gewähr für seine dauernde Erhaltung zu bieten. Vielleicht aber gibt es tiefer liegende Gründe, die zu der Hoffnung berechtigen, da 3 Deutschland sich nicht so leicht aus seiner günstigen Stellung, die es einnimmt, wird verdrängen lassen. Sollte das Volk der Denker und Dichter, wie man uns Deutsche wohl nicht ohne eine Beimengung von Geringschätzung genannt hat, nicht doch vielleicht in den vergangenen Jahrzehnten und Jahrhunderten gewisse geistige Eigenschaften und Fähigkeiten in sich entwickelt und ausgebildet haben, die sich bei den anderen Nationen nicht in gleichem Maße ausgeprägt finden, und die es ihm ermöglichen, sich in schwierige wissenschaftliche und technische Probleme mit einer Innigkeit zu vertiefen, die den Völkern mit impulsiverem Charakter, der sie zu böheren künstlerischen Leistungen befähigen mag, oder den Leuten des ,gesunden Menschenverstandes", der den politischen Erfolg verbürgt, nicht gegeben ist. Verfolgt man die Entwicklung, die gewisse Gebiete der Technik, und nicht nur der chemischen Industrie, in den letzten Jahrzehnten durcheilt haben, so wird man sich leicht davon überzeugen können, daß diese Entwicklung viel mehr nach der Tiefe als nach der Breite gestrebt hat. Es erscheint daher, wenn man von individuellen Erscheinungen und Erfahrungen auf analoge Verhältnisse bei ganzen Völkern oder Rassen schließen darf, durchaus nicht unmöglich, daß in nicht zu ferner Zukunft ein Zustand erreicht wird, bei dem das eine oder andere Land, auf Grund einer andersartigen Organisation seiner geistigen Kräfte, sich nicht mehr in der Lage sieht, in vollem Maße selbstschöpforisch an der Weiterentwicklung mitzuarbeiten. Es wird sich, nicht aus äußeren, sondern aus den oben angedeuteten inneren Gründen, statt wie bisher produktiv, nunmehr in der Hauptsache rezeptiv verhalten: Der Beginn einer allerdings nie streng durchzuführenden Arbeitsteilung unter den Völkern, entsprechend den jeweiligen Fähigkeiten.

Nicht nur die veränderten Verkehrsverhältnisse, die durch Dampf und Elektrizität geradezu eine Umwälzung erfahren haben, ermöglichen and erzwingen vielfach einen solchen erhöhten Austausch der Erzeugnisse; auch auf gesetzgeberischem Gebiete ist ein Umstand in hohem Maße geeignet, eine solche Entwicklung zu begünstigen und zu beschleunigen, ein Umstand, der auch bisher schon wesentlich dazu beigetragen hat, die Blüte der deutschen Teerfarbenindustrie zu fördern. Es ist das die Patentgesetzgebung und der in neuester Zeit durch sie herbeigeführte Zusammenschluß fast aller Kulturstaaten zu einem gemeinsamen Verbande zum
Schutz des gewerblichen Eigentums. Es würde zu weit führen, hier des Näheren die Wirkungon der Patentgesetzgebung und der eben erwähnten sogen. "Internationalen Union" auscinanderzusetzen. Es ist aber, wic vielfältige Erfahrung gelehrt hat, keine Übertreibung zu sagen, daß das gewerbliche Urheberrecht dem erfinderischen Geiste die Möglichkeit gewährt, seine Herrschaft über den ganzen Erdball auszudehnen und durch die Künste des Friedens Länder und Meere sich untertan zu machen.

Der gegenwärtige Zustand läßt die Hoffnung rege werden - und die oben angedeuteten inneren Gründe scheinen diese Hoffnung zu rechtfertigen -, daß aie Teerfarbenindustrie eines von den Cebieten menschlicher Tätigkeit sein wird, auf dem das Volk der Dichter und Denker bei der Teilung $\mathrm{nicht} \mathrm{zu}$ s pät gekommen ist.

Es liegt nahe, in diesem Zusammenhange auf einige Vorgänge wirtschaftlicher Natur hinzuweisen, die sich vor nicht langer Zeit im Schoße der Teerfarbenindustrie abgespielt haben, und die eineWiederholung ähnlicher Ereignisse auf anderen Gebieten der Volkswirtschaft darstellen. Ich meine den $\mathrm{Zu}$ sammensehluB mehrerer Teerfarbenfabriken zu einer Art Betriebsgomeinschaft. Es haben sich hier 2 Gruppen gebildet. Die eine umfalst die Farbwerke vorm. Meister Lucius \& Brüning in Höchst a. M. und die Firma Leopold Cassella \& Co. in Frankfurt a. M., die andere die Badische Anilin- und Soda-Fabrik in Ludwigshafen a. Rh., dic Farben. fabriken vorm. Friedr. Bayer \& Co. in Elberfeld und die A.-G. für Anilinfabrikation in Berlin. Dem Zusammenschluß war vorausgegangen eine Preisübereinkunft zwischen der ,Badischen" und den „Höchstern“" betreffend Indigo, von der weiter unten noch die Rede sein wird. Er hat zur Folge eine außerordentlich weitgehende Preisgabo der eigenen Selbständigkeit auf seiten der der Gemeinsehaft angehörenden Farbenfabriken, die die größten ihrer Art sind, und die Hunderte von Chemikern sowie Tausende von Arbeitern (über 20000 ) beschäftigen. Man wird nicht umhin können, die Größe des Opfers anzucrkennen, das einer als richtig erkannten Idce zu Tiebe gewagt wurde, und das jedenfalls dic $\mathrm{Fr}$ wartungen auch derer übersteigt, die einen genallen Einblick in die Eigenart dieser Riesenbetriebe besitzen. Ob der Zusammenschlu3, der erfolgte in der Absicht, die Erzeugung zu vereinfachen und zu verbilligen, um dadurch auch dem Wettbewerb des Auslandes wirksamer begegnen zu können, eine wirtschaftliche Notwendigkeit war, und ob er den Beteiligten den materiellen Vorteil bringen wird, den sie erhofft haben, muB dahingestellt bleiben. Auf alle Fälle haben die zusammengeschlossenen Gruppen ihre Stellung auf dem Weltmarkt auch dem Auslande gegenüber erheblich verbessert und gleichzeitig ihre Fabrikation und Terwaltung vereinfachen können. Auf der anderen Seite aber wird man sich, bei aller Bewunderung für die Größe des Beispiels, vor einer Überschätzung der von solchen Betriebsgemeinschaften zu erwartenden Wirkungen zu hüten haben.

\section{A) Roh- und Ausgangsmaterialien.}

Bezüglich der Roh- und Ausgangsmaterialien haben sich die Grundlagen der Teerfarbenfabrikation nicht wesentlich gcändert. Die Möglichkeit, die 
wichtigsten Kohlenwasserstoffe, Benzol, Naphtalin und Anthracen, weit über den Bedarf hinaus zu erzeugen, und der enge Zusammenhang mit der von Jahr zu .Tahr zu höheren Leistungen sich aufschwingenden Eisenindustrie entheben die Farbenfabriken der wichtigen Sorge, ob sie die Rohstoffe in genügender Menge herbeizuschaffen vermögen. Es ist darum auch fraglich, ob die Verarbeitung der russischen Petroleumrückstände auf aromatische Kohlenwasserstoffe, über die kürzlich sehr interessante Mitteilungen gemacht wurden (siehe Ref. riese Z. 18, 546 [1905]), jemals fïr Deutschland technische Bedeutung gewinnen wird. Unsere deutschen Destillationskokereien verbrennen Jáhr aus Jahr ein Tausende von Tonnen solcher wertvoller Bestandteile unter ihren Koksöfen, weil bei dem heutigen Preisstunde ihre Gewinnung nicht den genügenden Nutzen abwirft. Allerdings bieten die aus Petroleumrückständen erzeugten Kohlenwasserstoffe den nicht zu unterschätzenden Vorteil, daß sie vollkommen frei von Schwefel sind. Es hat sich nämlich bel näherer Untersuchung der von den deutschen Farbwerken verarbeiteten Benzole gezeigt, daß auch die reinsten, mehrfach durchfraktionierten Sorten desselben nicht ganz frei von Schwefel sind. (Vgl. die Referate in dieser Zeitschrift über S.Gehalt der Reinbenzole, die Methoden der Reinigung, über Thiophenbestimmung.)

Die durch den unvermeidlichen Gehalt des Teers an Wasser, das sich auf einfache Woise nicht entfernen läßt, bedingten Schwierigkeiten, die sich in starkem Stoßen, Überschäumen und dgl. äußorn und besonders im Anfang eine außerordentlich vorsichtige Destillation erfordern, haben $\mathrm{zu}$ versehiedenen Vorschlägen geführt, die aber, soweit sie Neues enthalten, praktisch nicht leicht durchführbar sein werden.

In neuerer Zeit scheint der Bedarf an Phenol und Kresol noch weiter gestiegen $\mathrm{zu}$ sein, sodaß einerseits die sogenannte synthetische Darstellung des Phenols aus Benzol durch Sulfonieren und Verschmelzen eine erhöhte Bedeutung erlangt hat, anderseits aber auch die Trenn ung der drei isomeren Kresole, die als $Z$ wischenprodukte für die Darstellung von Heilmitteln, Farb- und Sprengstoffen einen sehr unterschiedlichen Wert besitzen. Die synthetische Darstellung des Phenols verläuft bekanntlich in so befriedigender Weise, daß Verbesserungen wohl nur hinsichtlich einer möglichst rationellen Aufarbeitung der Sulfonierungs- und Schmelzprodukte möglich erscheinen. Im Gegensatz dazu bildet die einfache und scharfe Trennung der drei isomeren Kresole ein anscheinend noch nicht vollkommen gelöstes Problem. I)ie in den letzten Jahren in Patentschriften veröffentlichten Trennungs- und Reinigungsverfahren beruhen auf folgenden Reaktionen: 1. Darstellung der drei isomeren Kresoxacetsäuren (aus rohem Kresolalkali + Monochloressigsäure) und Trennung der Na-Salze auf Grund der verschiedenen Löslichkeit in Wasser. 2. Trennung von m- und p-Kresol (nach Entfernung des niedriger siedenden o-Kresols durch fraktionierte Destillation) durch Sulfonieren, Abscheidung der in Wasser schwer löslichen p-Kresolsulfonsäure oder ihres Na-Salzes und Abspaltung der Sulfongruppen. 3. Trennung des Gemisches aus $m$ - und p-Kresolsulfonsäure durch Abspaltung der Sulfongruppe nur aus der m-Kresolsulfonsäure mittels überhitzten Wasserdampfes bei $120-135^{\circ}$. 4. Behandlung des Gemisches von $\mathrm{m}$ - und p-Kresol mit wasserfreier Oxalsäure oder wasserfreien sauren Oxalaten, wobei sich der kristallinische saure Oxalester des p-Kresols, HOOC. $\mathrm{COOC}_{6} \mathrm{H}_{4} \cdot \mathrm{CH}_{3}$, (bzw. ein Salz desselben) bildet, der sich vom unveränderten $m-K r e s o l$ trennen läßt. 5. Einwirkung von Bisulfat auf das Gemisch von $\mathrm{m}$ - und $\mathrm{p}$-Kresol unter Bedingungen, unter denen sich lediglich die m-Kresolsulfonsäure bildet, während p-Kresol unverändert bleibt, alsdain Abtrennung der wasserlöslichen m-Kresolsulfonsäure von dem ölförmigen p-Kresol. f. Abscheidung des m-Kresols aus einem Gemisch von m- und p-Kresol in Form des neutralen, schwer löslichen m-Kresolcalciums mittels Ätzkalk. 7. Überführung des Kresolgemisches in die Doppelverbindungen aus Kresol und Kresolalkali z. B. von der allgemeinen Formel $3 \mathrm{ROH}+\mathrm{ROK}$ und Trennung durch fraktionierte Kristallisation.

\section{B) Zwischenprodukte.}

Bei der Darstellung von Zwischenprodukten handelt es sich vielfach weniger um die Auffindung neuer Substanzen, als vielmehr um die Verbesserung der bisherigen Methoden zu ihrer Gewinnung, d. h. um eine Verbilligung durch Steigerung der Ausbeute oder durch Verwendung wohlfeilerer Ausgangs- und Hilfsmaterialien oder um eine Erhöhung der Reinheit und dgl. Vielfach natürlich geben hier, wie auf vielen anderen Gebieten der Technik, patentrechtliche Verhältnisse den Anstoß zur Auffindung never Verfahren, eine Erscheinung, die durch die Besonderheiten unserer deutschen Patentgesetzgebung eine Erklärung findet. $\mathrm{DaB}$ übrigens das deutsche Patentgesetz trotz gewisser, besonders in letzter Zeit lebhaft erörterter Mängel, seine großen Vorzüge besitzt, nicht nur im Hinblick auf die Förderung wissenschaftlicher Erkenntnis, sondern auch für die Entwicklung der Technik, wird gern und allseitig anerkannt, und besonders die deutsche Teerfarbenindustrie hat allen Anlaß, dankbar des Schutzes eingedenk zu sein, den ihr Gesetz und Rechtsprechung verleihen.

Wegen ihrer großen Wichtigkeit und der Sonderstellung, die sie unter den Zwischenprodukten einnehmen, werden die Anthrachinonabkn̈mmlinge unter den Alizarinfarbstrffen (siehe Abschnitt C VIII) besprochen werden. In diesem Abschnitt sollen zunächst nur diejenigen Methoden Berücksichtigung finden, die sich auf Benzol- und Naphtalinderivate beziehen.

1. Di e Sulfoni erung. Diesse Methode, die einst, als es sich in den $80 \mathrm{er}$ und 90er Jahren. des vergangenen Jahrhunderts um den Ausbau des umfangreichen Gebietes der Naphtalinderivato handelte, vor allem in Verbindung mit der Alkalischmelze eine hervorragende Rolle in der Patentliteratur spielte, hat in den letzten Jahren bei der Darstellung von neuen Zwischenprodukten nur eine verhältnismäßig seltene Anwendung gefunden. Meist handelte es sich dabei um die Darstellung von Komponenten für Azofarbstoffe. Der umgekehrte Fall, nämlich der Verlust von Sulfongruppen, wurde bei der 1, 4, 6-, $1,4,7$ - und 1, 4. 8-Naphtylamindisulfonsäure beobachtet, die bei der Finwirkung aromatischer Amine (Anilin, Toluidin) unter Abspaltung der 4-ständigen 
Sulfongruppen in die arylierten $a$-Naphtylamin(6-, 7- und 8-)monosulfonsäuren übergehen. Diese Tatsache ist deshalb von Interesse, weil die Sulfongruppen in 4-Stellung in den $a$-Naphtylaminsulfonsäuren, im Gegensatz zu den $a$-Naphtolsulfonsäuren, in der Regel sehr fest sitzen.

2. Die Veranlassung zur Darstellung neuer $\mathrm{N}$ i trok ör p e r lag teils in den Schwefel-, teils in den Azofarbstoffen; im letzteren Fall schließt sich dann meist noch die Reduktion zu den entsprechenden Amidoverbindungen an. Eine bemerkenswerte Neuerung bei der Nitrierung von primären Aminen besteht darin, daß man als schützenden Acylrest, statt der bisher üblichen Formyl-, Acetyl-, Phtalylgruppen usw., einen Arylsulfonsäurerest, also z. B. $\mathrm{CT}_{3}, \mathrm{C}_{6} \mathrm{H}_{4}-\mathrm{SO}_{2}-$ einführt, der die gleiche Wirkung wie die rein organischen Acylreste ausübt, außerdem aber auch die Anwendung verdünnter Salpetersäure und die Erzeugung einheitlicher pbzw. o-Mononitroprodukte ermöglicht. Von Bedoutung ist das Verfahren auch für die m-Diamine, deren Di-Arylsulfonylverbindungen sich gleichfalls leicht in wässeriger Lösung nitrieren lassen. Die aus Eisessig oder Essigsäureanhydrid und stärkst konzentrierter Salpetersäure erhältliche Diacetylorthosalpetersäure, $\left(\mathrm{CH}_{3} \mathrm{COO}\right)_{2} \mathrm{~N}(\mathrm{OH})_{3}$, dürfte zum Nitrieren wohl nur in Ausnahmefällen Anwendung finden, wenn die in der Technik üblichen wohlfeileren Mittel versagen. Höchst interessante Beiträge zur Kenntnis der Nitriersäure (Mischung aus Salpetersäure und Schwefelsäure), die nicht nur in der Farben-, sondern auch in der Sprengstoffindustrie die ausgedehnteste Verwendung findet, lieferte $\mathrm{R}$ a s c h i g in einer Abhandlung (siehe diese Z. 18, 1281 ff. [1905]), die sich vorwiegend allerdings mit einem anderen Problem, der Theorie des Bleikammerprozesses, beschäftigt.

3. Die Reduktionsmethoden haben im abgelaufenen Jahrzehnt eine sehr eingehende Durcharbeitung erfahren, die man zurzeit in der Hauptsache wohl, wenigstens vorläufig, als abgeschlossen betrachten darf. Zahlreich sind die Bemühungen gewesen, durch Anwendung elektrolytischer Methoden den ReduktionsprozeB besser in der Hand und bei beliebigen $\mathrm{Z}$ wischenstufen ( $\mathrm{Az}$ oxy-, Hydroxylamin-, Azo-, Hydrazo-) festzuhalten. Wenn man die nicht unbeträchtliche Zahl der im angegebenen Zeitraum erteilten Patente mit den tatsächlichen Änderungen vergleicht, die die technische Darstellung der aus Nitrokörpern durch Reduktion erhältlichen Produkte erfahren hat, so wird man die technische Ausbeute, im Gegensatz zur wissenschaftlichen Bereicherung, nicht sonderlich hoch nennen können. Eine der wichtigsten Errungenschaften, die man dem Studium der Elektrolyse organischer Nitrokörper verdankt, ist wohl, außer der Verbesserung in der Gewinnung der Benzidin- usw. Basen, die Darstellung des als Ausgangsmaterial für die Schwefelfarbstoffe viel benutzten p-Aminophenols und seiner Derivate aus Nitrobenzol bzw. Phenylhydroxylamin; die als photographische Entwickler brauchbaren $\mathrm{p}$-Aminophenolderivate, der $\mathrm{p}$ Amidooxybenzylalkohol und die entsprechende Benzylsulfonsäure, scheinen jedoch leichter durch Reduktion der entsprechenden Nitrophenole erhältlich zu sein. Ein nicht zu unterschätzender Mangel, den alle derartigen Verfahren der elektrolytischen Re. duktion organischer Körper - und ähnliches gilt auch für die elektrolytische Oxydation derselben - aufweisen, ist der, daß 1 . bei Anwendung von Diaphragmen ein erheblicher Teil der Stromarbeit zur Uberwindung des Diaphragmenwiderstands verbraucht wird, und daß 2. bei der Übertragung der im Kleinen ausgezeichnet verlaufenden Prozesse ins Große gewisse Sehwierigkeiten entstehen, sobald es auf die richtige Bemessung von Stromstärke und -spannung ankommt. Gewöhnlich sind die Werte für Ampère und Volt an verhältnismäBig enge Grenzen gebunden, deren genaue Innehaltung die Apparatur unter (jmständen so kompliziert, daß alle die Vorteile, die - billige elektrische Energie vorausgesetzt - dem Verfahren an sich anhaften, durch dis Nachteile mehr wie aufgewogen werden. Mit berechtigter Spannung wird man dem Frgebnis der Versuche entgegensehen dürfen, die vor einigen Jahren erfundene außerordentlich sinnreiche Kombination der Alkalichloridelektrolyse mit der elektrolytischen Reduktion von Nitrokörpern (nach der G]eichung: $6 \mathrm{NaCl}+\mathrm{C}_{6} \mathrm{H}_{5} \cdot \mathrm{NO}_{2}+4 \mathrm{H}_{2} \mathrm{O} \rightarrow \mathrm{C}_{6} \mathrm{H}_{5} \cdot \mathrm{NH}_{2}+6 \mathrm{NaOH}$ $+6 \mathrm{Cl})$ der technischen Verwirklichung näher zu rücken. Vielleicht gelingt es diesem Verfahren, auf Grund der durch die obige Gleichung zum Ausdruck gelangenden günstigen Stromausbeute, sich das eine oder andere Gebiet zu erobern.

Eine gewisse Bedeutung wird einem in Frankreich erfundenen Verfahren zur Darstellung von Amido- aus Nitroverbindungen zugeschrieben. Auch abgesehen davon, daß nur solche Nitroverbindungen bei diesem Verfahren (das Wasserstoff oder Wassergas in Gegenwart gewisser Metalle $[\mathrm{Cu}, \mathrm{Ni}, \mathrm{Co}, \mathrm{Fe}$, Pt], die wie Katalysatoren wirken, als Reduktionsmittel benutzt) als Ausgangsmaterialien brauchbar sind, die, wie z. B. Nitrobenzol, leicht zu verdampfen sind, während Nitroverbindungen mit mehreren Substituenten, wie Di- und Trinitrokörper oder Nitrosulfonsäuren, davon ausgeschlossen bleiben, erscheint es fraglich, ob nicht auch dieses Verfahren, wie so viele andere an sich beachtenswerte Vorschläge; an der technischen Überlegenheit der seit Jahrzehnten auf das feinste durchgearbeiteten alten Reduktionsmethode, die auf der Verwendung der wohlfeilen Eisenfeil. oder Drehspäne beruht, scheitern wird.

4. Die $O x y d a t i o n s p r o z e s s e$ beziehen sich, im Anschluß an die Bemühungen zur Darstellung von o-Nitrobenzaldehyd für Indigo, vorwiegend auf die Überführung von $\mathrm{CH}_{\mathbf{3}}$ - in $\mathrm{COH}$ - und $\mathrm{COOH}$ - oder von $\mathrm{CH}_{2}<$ - in $\mathrm{CO}<$-Gruppen. Hierbei haben sich auch die Cerverbindungen als ausgezeichnete Kontaktsubstanzen oder Sauerstoffüber. trägger erwiesen. Die unmittelbare Hydroxylierung aromatischer Kohlenwasserstoffe $\mathrm{zu}$ den einfachsten Hydroxylverbindungen $\left(\mathrm{C}_{6} \mathrm{H}_{6} \rightarrow \mathrm{C}_{6} \mathrm{H}_{5} . \mathrm{OH}\right.$ oder $\left.\mathrm{C}_{10} \mathrm{H}_{8} \rightarrow \mathrm{C}_{10} \mathrm{H}_{7}, \mathrm{OH}\right)$, ein im Hinblick auf die bekannten Preisunterschiede sehr wichtiges tech. nisches Problem, hat bisher aus allerdings leicht verständlichen Gründen bezüglich des Benzols and Naphtalins noch keine Lösung gefunden. Die ein fache Übertragung der aus der Anthrachinonreihe bekannten Reaktionen ist wegen des durchaus ver schiedenartigen Verhaltens von Benzol und Naph talin gegen konzentrierte Schwefelsäure und Oleum nicht angängig. Die bereits vor einer Reihe von Jahren ausgeführte Hydroxylierung von Phenoler. 
mittels Persulfaten, die in der Regel in der p-Stellung stattfindet, entspricht keinem dringenden technischen Bedürfnis. Wichtiger, z. B. für Brenzkatechin und Guajakol, wäre ein Verfahren, die Hydroxy. lierung auch bei in p-Stellung nicht substituierten Verbindunger auf die o-Stellung zu beschränken. Mit Hilfe der Mn-Verbindungen $\left(\mathrm{MnO}_{2}\right.$ und Manganisalze) wurden neue Variationen gefunden zur Darstellung von o-Nitrobenzaldehyd, Benzaldehydsulfonsäuren, Chinonen und Carbonsäuren. Ein technisch aussichtsreiches Verfahren besteht in der Oxydation der Arylsulfonsäureester der Kresole, z. B. $\mathrm{CH}_{3} \cdot \mathrm{C}_{6} \mathrm{H}_{4} \cdot \mathrm{O} \cdot \mathrm{SO}_{2} \cdot \mathrm{C}_{6} \mathrm{H}_{5}$, zu Aldehyden, $\mathrm{H} . \mathrm{CO}$ . $\mathrm{C}_{6} \mathrm{H}_{4} .0 . \mathrm{SO}_{2} \cdot \mathrm{C}_{6} \mathrm{H}_{7}$, oder Säuren, HOOC. $\mathrm{C}_{6} \mathrm{H}_{4} . \mathrm{O}$ . $\mathrm{SO}_{2} \cdot \mathrm{C}_{6} \mathrm{H}_{5}$, die durch Verseifung in die freien Hydroxylverbindungen (Salicyl-Aldehyd oder -Säure) iibergehen.

In den letzten Jahren hat sich zu den bekannten Methoden eine neue eigenartige hinzugesellt, um Chlor-, Nitro-, Sulfon- und Alkoxygruppen durch Hydroxyl zu ersetzen. Es wird sich beim Abschnitt C II, Azofarbstoffe, Gelegenheit bieten, auf diesen Gegenstand zurückzukommen.

Bei den Chlorierungen, die in zahlreichen Fällen Anwendung gefunden haben, sowohl bei Farbstoffen als auch bei der Darstellung von Zwischenprodukten, wurde mehrfach statt des freien Chlors das Sulfurylehlorid, $\mathrm{SO}_{2} \mathrm{Cl}_{2}$, empfohlen.

Die in ihrer Mannigfaltigkeit unerschöpfliche Methode von Grignard hat für technische Zwecke bisher nur dort Anwendnng gefunden, wo es sich um wertvolle chemische Präparate, z. B. Riech- und Heilstoffe, handelte. Für die Teerfarbenindustrie hat das Verfahren zur Darstellung von Aldehyden aus Magnesiumhalogenalkylen(-arylen) und Ameisensäureester im Hinblick auf gewisse aromatische Aldehyde (z. B. o-Nitrobenzaldehyd) Interesse, falls der noch hohe Preis dos metallischen Magnesiums seiner technischen Verwendung nicht hindernd im Wege steht.

Im Anschluß hieran noch einige kurze Bemerkungen über die Neuerungen auf dem Gebiete der bei der Teerfarbenfabrikation benutzten Hiltsstoffe.

In der Entwicklung der Schwefelsäureindustrie ist, besonders was die Kontaktmethoden anlangt, ein gewisser Stillstand eingetreten, nachdem durch die vornehmlich von der Badischen Anilin- und Soda-Fabrik, den Höchster Farbwerken und dem Verein Chemischer Fabriken in Mannheim ausgebildeten Verfahren die Darstellung des $\mathrm{SO}_{3}$ eine hohe Stufe der Vollendung erreicht hat. Im Zusammenhang damit haben die Chlorsulfonsäure, $\mathrm{HSO}_{3} \mathrm{Cl}$ (aus $\mathrm{SO}_{3}+\mathrm{HCl}$ ), und das Sulfurylchlorid, $\mathrm{SO}_{2} \mathrm{Cl}_{2}$ (aus $\mathrm{SO}_{2}+\mathrm{Cl}_{2}$ ), als Hilfsmaterialien in neuerer Zeit mehrfache Verwendung gefunden, erstere für die Zwecke der Sulfonierung, die übrigens in gewissen Fällen einen anderen Verlauf nimmt als bei Anwendung von Schwefelsäure oder Oleum, letztere an Stelle des Chlors zum Chlorieren (s. o.), z. B. zur Darstellung von MTonochlorfettsäuron in Gegenwart ihrer Chloride bzw. Anhydride. Nicht ohne Interesse ist auch die Brauchbarkeit des chlorsulfosauren Natriums, $\mathrm{NaSO}_{3} \mathrm{Cl}$ (aus $\mathrm{NaCl}+\mathrm{SO}_{3}$ ), zur Darstellung von Acetylchlorid und Essigsäureanhydrid, nach den Gleichungen :

$$
\begin{gathered}
\mathrm{CH}_{3} \mathrm{COONa}+\mathrm{NaSO}_{3} \mathrm{Cl}=\mathrm{CH}_{3} \mathrm{COCl}+\mathrm{Na}_{2} \mathrm{SO}_{4} \\
2 \mathrm{CH}_{3} \cdot \mathrm{COONa}+\mathrm{NaSO}_{3} \mathrm{Cl}=\left(\mathrm{CH}_{3} \mathrm{CO}\right)_{2} \mathrm{O}+\mathrm{Na}_{2} \mathrm{SO}_{4} \\
+\mathrm{NaCl}
\end{gathered}
$$

Auf ähnlichen Reaktionen beruht die durch neuere Patente bekannt gewordene Darstellung von Säureanhydriden und Säurechloriden aus den Gemischen der Alkali- und Erdalkalisalze $+\mathbf{P C l}_{5}$ oder $\mathrm{POCl}_{3}$ oder $\mathrm{COCl}_{2}$.

Von großer Tragweite sind die Ergebnisse der Versuche, die in allerjüngster Zeit angestellt wurden behufs Erzeugung von Salpetersäure aus den Bestandteilen der Luft auf elektrischem Wege. Wenn auch die Landwirtschaft, die in Deutschland bisher etwa $4 / 5$ des gesamten aus Chile eingeführten Salpeters verbrauchte, vor allem Ursache hat, ïber die Erfolge zu frohlocken, die die von ihr nicht immer freundlich behandelte chemische Technik in heißem Bemühen errungen hat, Erfolge, dic für alle Zeiten die Menschheit der drohenden Gefahr einer Salpeternot entrücken, so darf auch die 'Teerfarbenindustrie, neben der Sprengstoffindustrie die Hauptabnehme. rin für Salpetersäure, getrost der Zukunft entgegensehen, ohne befürchten zu müssen, daß durch die allmähliche, früher oder später doch unvermeidliche Erschöpfung der chilenischen Salpeterlager die Preise für dieses unentbehrliche Hilfsmittel eine bedenkliche Höhe erreichen. Leider kann Deutschland nicht hoffen, an der Verwertung dieser Erfindung den selbsttätigen Anteil zu nehmen, den wir von ihm, wichtigen technischen Neuerungen gegen. über, gewöhnt sind. In diesem Falle sind bevorzugt die Länder mit ,flüssiger Kohle", deren potenzielle Energie, nach der schließlichen Umwandlung in elektrische Energie, den widerstrebenden Sauerstoff an den Stickstoff kettet; vor allem Skandinavien, die Schweiz und Italien. Für sie gewinnt das S c hiller sche: „Aus der Wolke quillt der Segen, strömt der Regen " einen neuen Tnhalt. Die Darstellung des für die Farbenindustrie unentbehrlichen Nitrits, die bisher stets auf dem Wege über den Salpeter erfolgte, wird von den neuesten Fortschritten wohl gleichfalls nicht unberiuhrt bleiben, da das bei der Vereinigung von $\mathrm{N}_{2}$ und $\mathrm{O}_{4}$ primär entstehende $\mathrm{N}_{2} \mathrm{O}_{4}$ durch Einleiten in Alkali in ein Gemisch von Nitrat und Nitrit übergeht, so daß es nur einer rationellen Trennung bedürfte.

Die übrigen Hilfsstoffe, wie $\mathrm{HCl}, \mathrm{Cl}$, die Oxy. dationsmittel $\mathrm{CrO}_{3}, \mathrm{MnO}_{2}, \mathrm{KMnO}_{4}$, die organischen Verbindungen Essigsäure, Acetylchlorid, Essigsäureanhydrid, Oxalsäure, Benzoylchlorid, Phtalsäureanhydrid usw., haben ihre bisherige Bedeutung bewahrt. Die Versuche, die billig gewordene Ameisensäure statt der Essigsäure zum Acylieren zu benutzen, wurden von verschiedenen Seiten fortgesetzt. Auch an dem Verfahren zur Darstellung von Oxalsäure aus Ameisensäure wurden weitere Verbesserungen erzielt durch Herabsetzung der zur Durchführung der Reaktion erforderlichen Menge Alkali (unter 5\%). Auf die neueren Untersuchungen über die Anwendbarkeit der Ameisensäure an Stelle der Wein- und Milchsäure beim Beizen der Wolle mit Chrom kann hier nur hingewiesen werden. $\mathrm{Zu}$ den mannigfaltigen Verwendungsarten, die der Formaldehvd seit einer Reihe von Jahren in der Farbenindustrie gefunden hat, ist in jüngster Zeit eine neue 
hinzugekommen, nämlich behufs Herstellung von Kondensationsprodukten mit der bisher als Hydrosulfit bezeichneten Verbindung $\mathrm{Na}_{2} \mathrm{~S}_{2} \mathrm{O}_{4}+2 \mathrm{H}_{2} \mathrm{O}$ und dem einen Spaltstück derselben, dem $\mathrm{NaHSO}_{2}$, Sulfoxvlat genannt. Es stchen somit zu Reduktionszweeken (d. h. zum Ätzen von Farbstoffen sowohI wie zum Verküpen) nunmehr drei ausgezeichnete Mittel zur Verfügung: 1. $\mathrm{Na}_{2} \mathrm{~S}_{2} \mathrm{O}_{4}$, das entwässerte Hydrosulfit, beständig gemacht durch Zusatz von Alkali und Glycerin. 2. Die Formaldehydverbindung desselben : $\mathrm{Na}_{2} \mathrm{~S}_{2} \mathrm{O}_{4}+2 \mathrm{CH}_{2} \mathrm{O}+2 \mathrm{H}_{2} \mathrm{O}=\mathrm{NaHSO}_{3}$ $+\mathrm{CH}_{2} \mathrm{O}+\mathrm{NaHSO}_{2}+\mathrm{CH}_{2} \mathrm{O}$, das Hydrosulfit $\mathrm{NF}$ der Höchster Farbwerke, und 3. die Formaldehydverbindung des im Hydrosulfit NF enthaltenen Spaltstückes: $\mathrm{NaHSO}_{2}+\mathrm{CH}_{2} \mathrm{O}$, der Rongalit $\mathrm{C}$ der Badischen Anilin- und Soda-Fabrik. Auf die höchst interessante Darstellung der verschiedenen Verbindungen, ïber die eine umfängliche Literatur besteht, (siehe Ref. diese Z. 18, 586, 587 [1905]), und die durch zahlreiche Patente geschützt sind, kann hier nicht näher eingegangen werden.

\section{C) Farbstoffe.}

Unter den Gegenständen von allgemeinerer Bedeutung haben die Konstitutionsfragen, ferner die Theorien über das Zustandekommen der Färbungen mittels organischer Farbstoffe, sowie die Theorie der Beizenfärbungen einen breiten Raum in don wissenschaftlichen Erörterungen eingenommen, ohne dab es auf einem dieser Gebiete zu einer völligen Klärung der Meinungen gekommen wäre. Vor allem sei bei dieser Gelegenheit auf die umfassenden Untersuchungen von $\mathrm{v}$ on $\mathrm{B}$ a e y e $\mathrm{r}$ hingewiesen, der durch die Einführung der "Carboniumvalenz" die Schwierigkeiten zu vermeiden sucht, die sich der Erklärung bestimmter Tatsachen und scheinbarer Widersprüche entgegensetzen. Über den Inhalt dieser Untersuchungen ist von mir in dieser Zeitschrift fortlaufend berichtet worden, und ich darf mich daher an dieser Stelle mit einem Hinweis begnügen.

\section{Triphenylmethanfarbstoffe.}

Der wundervolle, leuchtende Farbenton der Triphenylmethanfarbstoffe und ihre große Ausgiebigkeit machen es leicht begreiflich, daß die Farbstoffe dieser Klasse trotz ihrer in mehrfacher Beziehung ungenügenden Echtheitseigenschaften auch heute noch in gewaltigen Mengen Verwendung finden zum Färben solcher Waren, bei denen die Echtheit eine untergeordnete Rolle spielt, sondern wo es lediglich darauf ankommt, durch die Schönheit der Färbung eine vielfach allerdings nur vorübergehende Wirkung zu erzielen. Auf der anderen Seite aber hat man wohl aus den bisherigen Ergebnissen die Erkenntnis gewonnen, daß 3 von einem weiteren Ausbau dieses Gebiets für die Technik unter den heutigen Umständen nicht viel mehr zu erwarten ist, da man kaum hoffen darf, in dieser Klasse zu neuen Individuen zu gelangen, die allen gegenwärtigen Ansprüchen binsichtlich der Echtheit genügen, ohne durch zu hohen Preis die technische Verwendung auszuschließen. Die wesentlichsten bisherigen Fortschritte bezogen sich auf die Verbesserung der Alkaliund der Waschechtheit der Triphenylmethanfarbstoffe; von geringerem Erfolg waren die Bemühungen, lichtechte Produkte zu erzeugen. Besonders nachdem es gelungen ist, unter den Anthrachinonab. kömmlingen solche zu finden, die, wie z. B. Chinizaringrün und Alizarinsapbirol, leuchtenden Ton mit Fehtheit gegen Licht, Alkali, Seife und Walke verbinden, und die infolge ihrer verhältnismäßig einfachen Darstellungsweise einen mäßigen Preis gestatten, läBt sich woh] behaupten, daß das Streben nach echten Triphenylmethanfarbstoffen einem dringenden Bedürfnis nicht mehr entspricht.

Die bisherigen Annahmen über die Abhängigkeit des Reaktionsverlaufs vom Lösungs- und Kondensationsmittel bei der Farbstoffsynthese aus $\mathrm{Hy}$ drolen und Derivaten des o- und p-Toluidins haben eine erneute Bestätigung gefunden bei der Darstellung von Farbstoffen aus Tetraalkyldiamidohydrolen und Dialkyl-p-toluidinen. Der Unterschied tritt sogar bei den Dialky]-p-toluidinen, offenbar infolge des einheitlicheren Verlaufs der Kondensation, noch klarer hervor als bei den Monoalkylderivaten oder gar bei der primären Base selbst, bei der die richtungsbestimmenden Einflüsse noch um die Vorherrschaft kämpfen. Übrigens dürfte die in Patentschriften und neuerdings auch. von Friedländer (Bd. \%, S. 135) vertretene Ansicht, daß bei der Kondensation von $\mathrm{Hy}$ drolen mit 1,3-Naphtolsulfonsäuren (bzw. deren Äthern) Produkte entstehen, in denen sich die OHGruppe in p-Stellung zum Methan-C befindet, sich bei näherer Prüfung kaum als zutreffend erweisen. Bei der Darstellung von Azofarbstoffen aus 1,3Naphtolsulfonsäuren ist es schon lange und mit Sicherheit bekannt, daß die Azogruppe in o-Stellung zum Hydroxyl tritt. Neuerdings ist eine analoge Erscheinung zutage getreten in einem Falle, der über den Reaktionsverlauf keinen Zweifel übrig läßt, nämlich bei der Kondensation der sogenannten Rhodaminzwischenprodukte (p-Dialkylamido-o-oxybenzoylbenzoesäuren) mit Phenolen und Naphtolen zu Rhodinen und Rhodolen. Es ist in hohem Maße bemerkenswert, daß das unsubstituierte $a$-Naphtol fast ausschließlich und das gewöhnliche Phenol wenigstens teilweise das zur OH-Gruppe o-ständige $\mathrm{H}$-Atom unter Bildung eines Xanthenringes reagieren lassen. Im Einklang steht die Tatsache ferner mitder Entstehung der 0-Oxynaphtoylbenzoesäure bei der Kondensation von Phtalsäureanhy. drid mit $\alpha$-Naphtol und der schon vor Jahren von O. F i s c h or und Frän kel ausgeführten Synthese des o-Amidomalachitgrïns aus $\mathrm{Mi}$ ch $\mathrm{l}$ er schem Hydrol und Anilin. Da die aus 1,3-Methoxynaphtalinsulfonsäure entstehenden Produkte mit den aus 1,3-Naphtolsulfonsäuren durch nachträgliche Alkylierung entstehenden identisch sind, so ist anzunehmen, daß anch im ersteren Falle das in 2-Stellung befindliche H-Atom mit dem Hydrol in Reaktion tritt.

Interessant sind die in Wasser leicht löslichen ungefärbten Fuchsinpräparate, dıe aus Fuchsin durch Behandlung mit konz. Salzsïure in der Kälte erhalten werden können, und die dem bisweilen störenden Mangel der Schwerlöslichkeit des gewöhnlichen Fuchsins abhelfen sollen. In der Gruppe der A u r a mine wurden N-Alkyl-(Äthyl-) und -Aralkyl-(Benzyl-)derivate dargestellt, die vor dem gewöhnlichen Auramin den Vorzug größerer Seifenund Sodaechtheit besitzen sollen. 


\section{II. $\mathrm{Az}$ of $\mathrm{arbs}$ of $f \mathrm{e}$.}

DieAzofarbstoffe bilden eine Klasse, die trotz des Indigos, der Alizarin- u. Schwefelfarbstoffe auch heute noch von der allergrößten technischen Bedeutung ist, wenn auch, wie sich dies leicht voraussagen ließ, die Erfindungen, besonders im Bereich der Schwefelfarbstoffe, nicht ohne tiefgreifenden Einfluß auf sie geblieben sind. Aber die außerordentliche Vielseitigkeit sowohl der Darstellung als auch vor allem der Verwendung, die diese Farbstoffklasse vor allen anderen auszeichnet, sorgt dafür, daB für verloren gegangene Provinzen sich immer wieder ein Ersatz findet. Vor allem auffallend, aber wohl verständlich ist der Rückgang der Erfindungen, die sich auf die Darstellung von Polyazofarbstoffen für Baumwolle beziehen. Während in früheren Jahren eine unübersehbare Fülle von Patenten erkennen ließ, mit welchem Eifer die weitere Ausbildung dieses wichtigen Gebiets erstrebt wurde, findet sich gegenwärtig nur wenig, was einer besonderen. Beachtung wert erscheint. Meist handelt es sich nicht so sehr um neue Gesichtspunkte als vielmehr um die Benutzung neuer Azo- und Diazokomponenten, wie z. B. das Acetyltriamidobenzol, $\mathrm{CH}_{3}$. CO. $\mathrm{NH}: \mathrm{NH}_{2}: \mathrm{NH}_{2}$ $=1: 2: 4$, das Glycin der J-Säure, HOOC. $\mathrm{CH}_{2} . \mathrm{NH}$ : $\mathrm{OH}: \mathrm{SO}_{3} \mathrm{H}=2: 5: 7$, der p-Diamidohydrochinondialkyläther bzw. das Acetylderivat desselben oder der entsprechende Nitroamidokörper usw.

Eine bemerkenswerte Klasse von substantiven, auf der Faser weiter entwickelbaren Monoazoverbindungen stellen diejenigen Baumwollfarbstoffe dar, die auf folgendem Wege gewonnen werden: 1. Kombination von $m$ - oder p-Nitroanilin oder -toluidin mit einer geeigneten Naphtylaminsulfonsäure zu einem o-Amidoazofarbstoff, 2. Oxydation desselben zur Triazoldisulfonsäure, z. B.

$$
\left(\mathrm{NaO}_{3} \mathrm{~S}\right)_{2} \mathrm{C}_{10} \mathrm{H}_{4}<\frac{\mathrm{N}}{\mathrm{N}}>\mathrm{N} \cdot \mathrm{C}_{6} \mathrm{H}_{4} \cdot \mathrm{NO}_{2},
$$

3. Reduktion der Nitro- zur Amidoverbindung und 4. Verschmelzung zur Oxytriazolsulfonsäure,

$$
\mathrm{NaO}_{3} \mathrm{~S}>\mathrm{C}_{10} \mathrm{H}_{4}\left\langle\underset{\mathrm{N}}{\mathrm{HO}}>\mathrm{N}-\mathrm{C}_{6} \mathrm{H}_{4}-\mathrm{NH}_{2}\right. \text {. }
$$

Diese läßt sich mittels einer Diazoverbindung überführen in den Körper

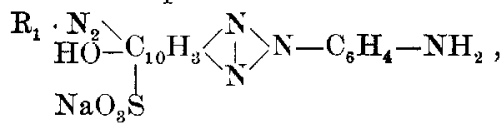

und nach dem Ausfärben wird dieser Monoazofarbstoff weiter entwickelt auf der Faser zum Disazofarbstoff

$$
\left.\underset{\mathrm{NaO} S}{\mathrm{HO}-\mathrm{C}_{10} \mathrm{H}_{3}} \underset{\mathrm{N}}{\mathrm{R}}\right\rangle \mathrm{N} \cdot \mathrm{C}_{\mathrm{i}} \mathrm{H}_{4} \cdot \mathrm{N}_{2} \cdot \mathrm{R}_{2} .
$$

(Rosanthrene).

Nahe verwandt mit den obigen Monoazofarbstoffen sind die z. B. aus Nitrobenzoylamidonaphtolsulfonsäuren,

$$
\underset{\mathrm{HO}_{3} \mathrm{~S}}{\mathrm{HO}}>\mathrm{C}_{10} \mathrm{H}_{5} \cdot \mathrm{NH} \cdot \mathrm{CO} \cdot \mathrm{C}_{6} \mathrm{H}_{4} \cdot \mathrm{NO}_{2},
$$

in leicht ersichtlicher Weise zu gewinnenden Farbstoffe von der Konstitution

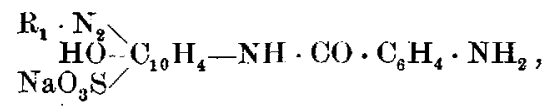

die sich gleichfalls nach dem Ausfärben weiter entwickeln lassen zu Disazofarbstoffen der allgemeinen Formel

$$
\begin{aligned}
& \mathrm{R}_{1} \cdot \mathrm{N}_{2} \backslash \mathrm{C}_{10} \mathrm{H}_{4} \cdot \mathrm{NH} \cdot \mathrm{CO} \cdot \mathrm{C}_{6} \mathrm{H}_{4} \cdot \mathrm{N}_{2} \cdot \mathrm{R}_{2} \\
& \mathrm{NaO}_{3} \mathrm{~S}
\end{aligned}
$$

von ähnlichen Echtheitseigenschaften wie die Triazolderivate. Bei den aus J-Säure dargestellten Monoazofarbstoffen der Formel

$\mathbf{R}_{\mathbf{1}} \cdot \mathbf{N}$

$$
\mathrm{HO}_{2}-\mathrm{O}_{10} \mathrm{H}_{4} \cdot \mathrm{NH} \cdot \mathrm{CO} \cdot \mathrm{NH} \cdot \mathrm{C}_{6} \mathrm{H}_{4} \cdot \mathrm{NH} \cdot \mathrm{OO} \cdot \mathrm{OH}_{3}
$$
$\mathrm{NaO}_{3} \mathrm{~S}$

gelangt die schon aus zahlreichen älteren Patentschriften bekannt gewordene Eigenschaft der $\mathrm{J}$. Säurederivate, substantive Monoazofarbstoffe (Be n zoe c htfarben-B a yer) zu bilden, emeut zur Anwendung. Die letzterwähnten Farbstoffe, die, wie man sieht, den Übergang zu den oben angefïhrten Entwicklerfarbstoffen bilden und wohl offenbar auch den Anstoß zu deren Darstellung gegeben haben, können übrigens gleichfalls in der üblichen Weise auf der Faser gekuppelt werden. Nahe verwandt mit den Triazolverbindungen sind ferner auch die aus den Amidothiazolbasen und Nitrobenzoylchlorid durch weitere Reduktion erhaltenen Amidobenzoylamidothiazole und deren Sulfon säuren, die gleichfalls als Entwicklerfarbstoffe anwendbar sind. Die an sich farblosen Thiazolsulfonsäuren wurden neuerdings dargestellt aus den Benzyliden- und Benzylderivaten von Naphtylaminsulfonsäuren oder Amidonaphtolsulfonsäuren durch die Einwirkung von Polysulfiden bei höheren Temperaturen, z. B.

$$
\begin{aligned}
& \underset{\mathrm{HO}}{\mathrm{HO}_{3} \mathrm{~S}}>\mathrm{C}_{10} \mathrm{H}_{5} \cdot \mathrm{N}=\mathrm{CH} \cdot \mathrm{C}_{6} \mathrm{H}_{5} \\
& +\mathrm{S}-\mathrm{H}_{2} \underset{\mathrm{HO}}{\mathrm{HO}}>\mathrm{C}_{10} \mathrm{H}_{4}\langle\mathrm{~S}\rangle \mathrm{C} \cdot \mathrm{O}_{6} \mathrm{H}_{5} \text {. }
\end{aligned}
$$

Die den S- entsprechenden $\mathrm{O}-$ Verbindungen sind die Naphtoxazole, die nach dem Schema

$$
\begin{aligned}
& \underset{\mathrm{HO}}{\mathrm{HO}_{3} \mathrm{~S}}>\mathrm{C}_{10} \mathrm{H}_{4}<\mathrm{OH}^{\mathrm{N}}+\mathrm{HOOC} \cdot \mathrm{C}_{6} \mathrm{H}_{5} \\
& \begin{array}{r}
\mathrm{HO}_{3} \mathrm{~S} \\
\mathrm{HO}
\end{array} \mathrm{C}_{10} \mathrm{H}_{4}\left\langle\mathrm{~N}>\mathrm{C} \cdot \mathrm{C}_{6} \mathrm{H}_{5}+2 \mathrm{H}_{2} \mathrm{O}\right.
\end{aligned}
$$

aus Amidodioxynaphtalinsulfonsäuren gewonnen wurden und in analoger Weise wie die Thiazole und Amidothiazole zur Farbstoffdarstellung sich verwenden lassen.

Die technische Bedeutung aller dieser neueren Entwicklerfarbstoffe liegt darin, daßB sie im Gegensatz zu den älteren Farbstoffen dieser Art, unter denen die sogenannten Diaminfarbstoffe, z. B. :

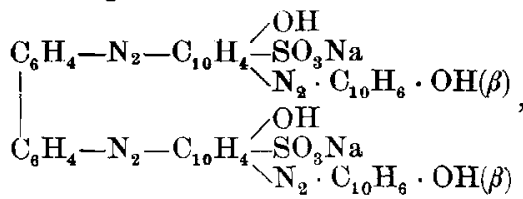

die wichtigsten sind - statt der dunklen, schwarzen, blauschwarzen und blauvioletten - helle, orange und bläulichrote Töne aufweisen und damit eine Lücke ausfüllen, die trotz der Schwefelfarbstoffe es dem Baumwollfärber bisher nicht ermöglicht hatte, auf billige Weise echte Färbungen der genannten Tonart zu erzielen.

Von großem technischen Interesse sind auch die Fortschritte, dio in letzter Zeit auf dem Gebiete 
der einfachsten Eisfarben erzielt worden sind. Die Anwendbarkeit des an sich sehr einfachen Verfahrens zur Erzeugung von brauchbaren Färbungen auf Baumwolle durch Kombination einer Diazoverbindung mit einer Azokomponente auf der Faser war bedingt einerseits natürlich durch die Echtheitseigenschaften (vor allem auch Sublimierechtheit), andererseits aber auch durch die Möglichkeit, in einfacher Weise durch Atzungen Weiß- und Buntcffekte hervorzubringen. Nachdem diese letzteren Schwierigkeiten, wie schon früher erwähnt, durch die weitere Vervollkommnung der Hydrosulfitätzmethoden als beseitigt gelten können, hat man neuerdings auch die schwerer ätzbaren Nuancen auf Baumwolle mittels des genannten Verfahrens erzeugt, wie z. B. das $a$-Naphtylamingranat oder -bordeaux und das Chrysoidinpuce (aus Chrysoidin + p-Nitrobenzoldiazoniumchlorid), das als Disazofarbstoff auch Halbätzen zuläßt, ferner das Rotbraun aus $p$-Nitranilindiazo-o-toluidin $+\beta$-Naphtol sowie das Echtgranat aus m-Amidoazotoluol.

Die nachchromierbaren Azofarbstoffe auf Wolle haben ihre große Bedeutung nicht nur beibehalten, sondern es ist sogar möglich gewesen, ihre Wichtigkeit noch zu erhöhen durch Auffindung neuer Diazo- und Azokomponenten, wie z. B. die o, o-Diamidophenol-p-sulfonsäure, die o,o-Diamido-1chlorbenzolsulfonsäure, die 1,3-Diamido-4-chlorbenzol-5-sulfonsäure, die m-Phenylendiamindisulfonsäure einerseits, die Triamidobenzole und deren Acetylverbindungen, die Diox ynaphtaline $(1,5 ; 1,6$; $1,7 ; 2,6)$ und Amidonaphtole $(1,5 ; 2,6 ; 2,7)$ sowie ihre Sulfonsäuren, ferner p-Kresol, die m-Amidophenole (-Kresole), m-Oxydiphenylamin, Acidyl-paminophenol usw. andererseits. Zwei Frfindungen der letzten Jahre, die gleichzeitig auch wissenschaftliches Interesse boanspruchen, seien hier besonders erwähnt. Sie beziehen sich beide auf die Darstellung der wichtigen o-Oxvazofarbstoffe. Die ältere beruht auf der Erkenntnis, daß Diazoverbindungen, in denen sich die Sulfon-, Nitro- oder Alkoxygruppe oder ein Halogen in o-Stellung zur Diazogruppe befindet, sehr starke Neigung zeigen, unter der Einwirkung von säturebindenden Mitteln wie Acetat, Bicarbonat, Soda, Magnesia und dgl, in o-Oxydiazoverbindungen überzugehen, in denen $\mathrm{SO}_{3} \mathrm{H}, \mathrm{NO}_{2}$, $\mathrm{H}$ alogen usw. durch $\mathrm{OH}$ ersetzt ist. Durch Hinzufügen von Oxydationsmitteln wie $\mathrm{Cl}$ oder $\mathrm{H}_{2} \mathrm{O}_{2}$ läßt sich die hierbei aus der Diazosulfonsäure entstehende $\mathrm{SO}_{2}$ unschädlich machen. Diese Erfindung ermöglicht z. B. die Darstellung dor 2-Diazo-1-naphtol-6-sulfonsäure aus der 2-Naphtylamin-1,6-disulfonsäure oder der 2-Naphtylamin-1-chlor-6-sulfonsäure. Die gleichen Diazonaphtolsulfonsäuren lassen sich auf einem anderen Wege, nach der 2 . hier zu erwähnenden Reaktion gewinnen. Die früheren Versuche, 0-Amidonaphtol-(1, 2- und 2,1-)sulfonsäuron zu diazotieren, waren bisher an dem anormalen Verlaut des Prozesses, den man der leichten Zersetzlichkeit der entstandenen Diazonaphtolsulfonsäuren zuschrieb, gescheitert. Daß diese aber tatsächlich gar nicht so leicht zersetzlich sind, wie man früher vermutete, wird bewiosen durch die Möglichkeit, sie mittels des gewöhnlichen Nitriergemisches zu nitrieren. Bozüglich der 1,2-Amidonaphtolsulfonsäuren wurde gefunden, daß bei Anwendung von organischen statt der Mineral-Säuren die Diazotie- rung wesentlich glatter und rascher verläuft. Auffallenderweise besitzen die so erhältlichen Diazo. verbindungen ein verhältnismäßig geringes Kupplungsvermögen, indem sie nur mit dem reaktionsfähigen Resorcin und auch mit diesem nur in alkalischer Lösung zu regulären Azofarbstoffen, nicht aber mit $a$ - oder $\beta$-Naphtol zusammentreten. Von noch größerer Bedeutung ist die Beobachtung, daß die normale Einwirkung der salpetrigen Säure auf 1,2- und 2,1-Amidonaphtolsulfonsäuren erheblich begünstigt wird durch die Gegenwart von metallischem Kupfer oder dessen Salzen. Da die oAmidonaphtolsulfonsäuren leicht zugïnglich sind (durchReduktion entweder derNitrosonaphtolsulfonsäuren oder der entsprechenden o-Oxyazofarbstoffe), so bedeutet diese neue Variation der Diazotierung eine wichtige Bereicherung der zur Darstellung von nach chromierbaren o-Oxyazofarbstoffen verfügbaren Diazoliomponenten, unter denen bisher fast ausschließlich die Derivate des o-Amidophenols zur Verwendung kommen konnten.

Den Schwierigkeiten beim Färben mit dem schwer löslichen Säurealizarinschwarz (o-Diamidophenol-p-sulfonsäure[tetrazotiert] $+2 \mathrm{Mol} . \beta$-Naphtol), das wegen seiner Lichtechtheit auch zum Färben von Militärtuchen zugelassen wurde, hat man durch Behandlung des Farbstoffes mit Bisulfit und darauf mit Mineralsäuren erfolgreich abgeholfen.

Unter den direkt auf Beizen ziehenden Wollazofarbstoffen sind in neuerer Zeit auch vielfach gemischte Disazofarbstoffe, die sich von Diaminen, wie Benzidin oder Phenylendiamin ableiten, und den Salicylsäure- (bzw. Kresotinsäure-) Rest enthalten, vorgeschlagen worden.

Es ist im Hinblick auf die in der Regel wenig befriedigenden Echtheitseigenschaften der gewöhnlichen sauren Wollazofarbstoffe, bei denen insbesondere die Seifen-, Walk-, Wasser- und Pottingechtheit zu wünschen übrig läßt, nicht $z u$ verwundern, daß sie gegenüber den wesentlich echteren nachchromierbaren Azofarbstoffen, deren Darstellung sowohl als auch Anwendung (im Einbadverfahren) sich höchst einfach gestaltet, ein wenig ins Hintertreffen gekommen sind, was sich sehr deutlich auch in der Patentliteratur ausprägt. Was den neveren unter ihnen noch zu einigem Interesse verhilft, das sind die besonderen Verwendungsarten, z. B. im Wolldruck, oder ihr besonderer Farbenton (gelb oder grünstichig blau) und ein gutes Egalisierungsvermögen, Bedingungen ihrer Brauchbarkeit, die besonders von den Chromotropfarbstoffen (neuerdings auch aus Chlorchromotropsäure) mehr oder minder weitgehend erfüllt werdon.

Auch auf einem anderen für die Azofarbstoffe von Jahr zu Jahr wichtiger werdonden Gebiete macht sich das Streben nach erhöhter Echtheit geltend. Das sind die Lackfarben. Es handelt sich hier in der Regel um einfache Monoazofarbstoffe, zu denen als Diazokomponenten außer den gewöhnlichen aromatischen Aminen der Benzol- und Naphtalinreihe, sowie ihren Sulfon- und Carbonsäuren und Ätherderivaten, mit Vorliebe ihre Nitro- und Chlorsubstitutionsprodukte ( $p$-Nitranilinsulfonsäure, o-Nitranilin-p-sulfonsäure, Mono- und Dichloranilinsulfonsäure, Chlornitramlinsulfonsäure, Trichloranilin, Nitrotoluidin) Verwendung finden und als Azokomponenten, außer $\beta$-Naphtol und den gewöhn- 
lichen Naphtolsulfonsäuren, die $\beta$-Oxynaphtoesäure, Nitro-m-phenylendiamin und dessen Disulfonsäure, für gelbe Lacke die Phenylmethylpyrazolone, die in letzter Zeit, wohl infolge der Verbilligung des Acetessigesters, in verstärktem Maße als Azokompo. nenten benutzt werden, ebenso wie auch die Indole. Die Anforderungen, die an derartige Lacke gestellt werden, sind außerordentlich mannigfaltig, je nach dem Zweok, dem sie dienen sollen, ob zum Anstrich von Holz oder zur Herstellung von öl- oder spritlöslichen Farben, oder zum Buch- und Steindruck, oder für die Tapeten- und Papierfärberei und dgl. Die schon seit längeren Jahren aus den gewöhnlichen Ponceaux gebildeten Lacke erweisen sich in der Regel nicht als genügend lichtecht, was z. B. bei ihrer Verwendung im Tapetendruck sehr störend hervortritt. Die in neuerer Zeit hergestellten Lacke weisen in dieser Richtung wesentliche Fortschritte auf, so daß die Teerfarbstoffe wie im Zeugdruck, so auch im Buch-, Stein- und Tapetendruck die anorganischen Verbindungen, deren Anwendung in hygienischer Hinsicht bisweilen nicht unbedenklich erscheint, zu verdrängen beginnen.

(Schluk folgt.)

\section{Praktische Beiträge zur Bleikammer= theorie.}

\section{Von Fabrikdirektor Dr. Siegmdnd L.jttmana.} (Eingeg. d. 17./4. 1906.)

Anläßlich Ausschaltung einer Hauptkammer behufs Reparatur derselben, oblag mir ungefähr vor Jahresfrist die Pflicht, den weiter arbeitenden Teil des Systems nach Möglichkeit produktiv zu gestalten. Die Gelegenheit kam mir auch willkommen, um eine Reihe von längst projektierten Versuchen auszuführen, über welche ich teilweise bereits in meinen jüngst veröffentlichten Studien über das ,Verhaltendes Selens im Sohwefel. s äurebetrie be" ${ }^{\text {"1) }}$ Mitteilungen machte. Gewissermaßen als Fortsetzung derselben soll nun auch über die Resultate einer Versuchsreihe zur Beurteilung der Bleikammerreaktionen $\mathrm{s}$ clbst berichtet werden. Diese war bereits vor Jahresfrist in dem hier dargelegten Maße vollständig beendigt; ich zögerte mit der Publikation, da ursprünglich eine allgemeine Erweiterung geplant war, woran mich jedoch Berufstätigkeit verhinderte. - Obwohl sich nun die Versuche nur im bescheidenen Rahmen bewegen, und deren Ergebnisse auch von bereits bekannten Tatsachen kaum abweichen, erschien mir eine Veröffentlichung doch nicht für überflüssig : müssen ja bedauerlicherweise noch so manche Widersprüche entwirrt wer. den, bei welchen es dem Praktiker oft ganz unerklärlich erscheint, warum bezüglich so einfacher Fragen die Theorie noch immer kein unanfechtbares Positivum, ja nicht einmal annäherungsweise eine Norm schaffen konnte.

Von ausführlichen Literaturangaben habe ich abgesehen, da ja diesbezüglich nahezu verschwende-

1). Diese Z. 19, 1039 (1906).

2) Z. physikal. Chem. 47, 513 (1904). Ch. 1906. risch ausgestattete Werke zur Verfügung stehen (L u n g e, Handbuch der Schwefelsäurefabrikation, T r a u t $z^{2}$, Zur physikalischen Theorie des Bleikammerprozesses).

Der von mir eingeschlagene Weg war der, daß ich an zahlreichen bestimmten Orten e i n u n d d e s selben Längsschnittes im Innern einer Hauptkammer, aber unter vers chiedensten Betriebsbedingungen, die daselbst gebildete Säure in Glasgefäßen auffing. L unge hält diese Beobachtungsweise für verfehlt. Die hier mitgeteilten Ergebnisse sollen selbst dafür sprechen, ob sie wirklich wertlos ist zur Beurteilung der Reaktionen in den Kammern, oder ob etwa dieser Weg bei zielbewußter Anordnung nicht weitgehenderen Einblick gestattet und auch einwandfreier ist, als Gasanalysen und Temperaturbestimmungen allein. Allerdings könnte man leicht auf Irrwege geraten oder zu ganz entgegengesetzten Resultaten kommen (H. A. S m i th, M a c te a r usw.), wenn man etwa aus der aufgefangenen Säuremenge und dem Nitrosegehalt a 11 e in Schlüsse ziehen wollte, ohne entsprechende Kontrolle und Heranziehung sonstiger qualitativer und quantitativer Beobachtungen, hauptsächlich aber $\mathrm{f} a \mathrm{k}$ tischer Betriebsresultate der unter verschiedenen Bedingungen arbeitenden Kammer.

Um langwierige Beschreibungen der Versuchsreihe zu umgehen, möchte jch beistehende Skizzen zu Hilfe nehmen und gleichzeitig für die später folgenden Diagramme einige Bezeichnungen einführen.

$a(1: 2)$

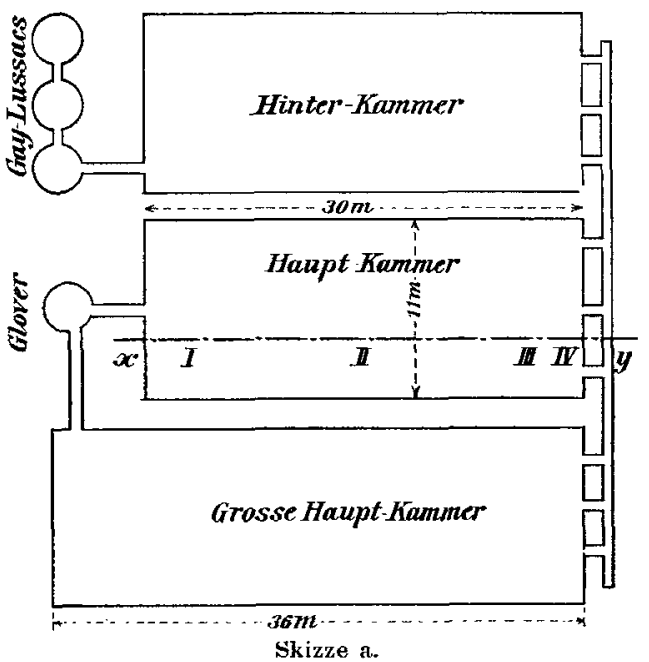

Skizze a zeigt die Anordnung des Gesamtsystems. Hiervon ist die Mittelkammer als Versuchskammer benutzt worden. Die Kammern sind. wohl etwas breiter $(11 \mathrm{~m})$, als man sie zurzeit baut, zeigen aber sonst nichts Anormales. Die Versuchskammer $(7 \times 30 \times 11 \mathrm{~m}=2300 \mathrm{cbm})$ ist genau so groß, wie die darauf folgende Hinterkammer. Für unsere Versuche kommt eigentlich n u r d i e ses Zweikammersystem in Betracht; allerdings ist in diesem Falle die Hinterkammer etwas zu groß. Es sind drei große, gut arbeitende Gay-Lussacs mit Koksfüllung vorhanden, also in jeder Beziehung $\mathrm{m}$ e h $\mathrm{r}$ als ausreichend. 